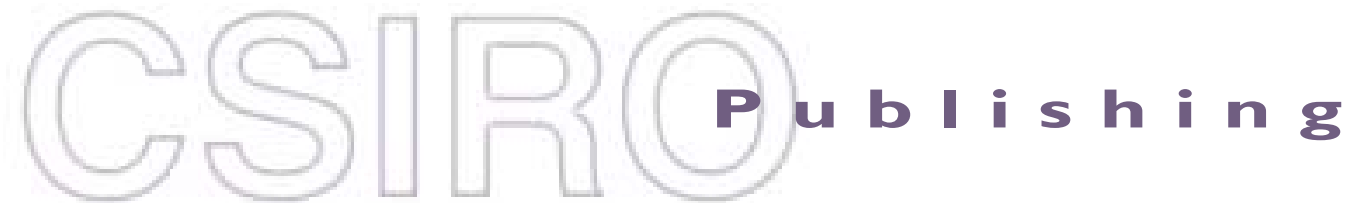

\section{Publications of the Astronomical Society of Australia}

Volume 19, 2002

(C) Astronomical Society of Australia 2002

An international journal of astronomy and astrophysics

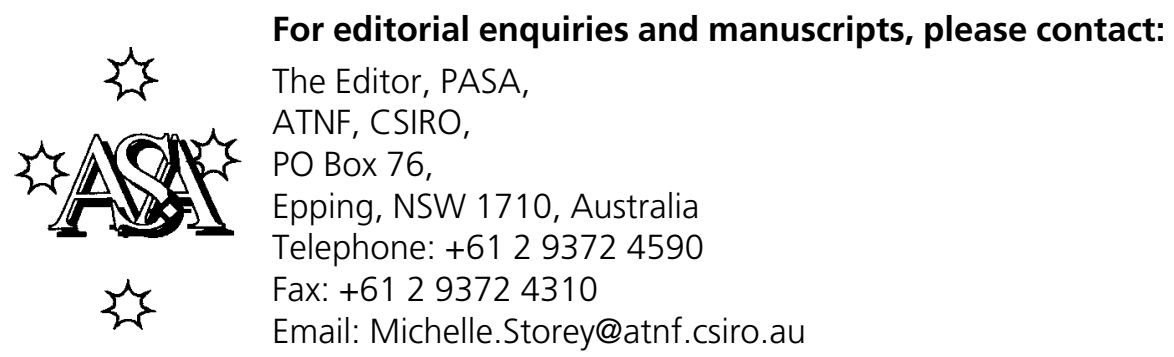

For general enquiries and subscriptions, please contact: CSIRO Publishing PO Box 1139 (150 Oxford St)

Collingwood, Vic. 3066, Australia

Telephone: +6139662 7666

Fax: +61 396627555

Email: publishing.pasa@csiro.au

C S I RO

PUBLISHING Published by CSIRO Publishing

for the Astronomical Society of Australia

www.publish.csiro.au/journals/pasa 


\title{
The Potential of Differential Astrometric Interferometry from the High Antarctic Plateau
}

\author{
James P. Lloyd ${ }^{1}$, Ben R. Oppenheimer ${ }^{2}$ and James R. Graham ${ }^{1}$ \\ ${ }^{1}$ Department of Astronomy, University of California, Berkeley, USA \\ jpl@astron.berkeley.edu \\ ${ }^{2}$ Department of Astrophysics, American Museum of Natural History, New York, USA \\ Received 2001 August 1, accepted 2002 February 7
}

\begin{abstract}
The low infrared background and high atmospheric transparency are the principal advantages of Antarctic Plateau sites for astronomy. However, the poor seeing (between 1 and 3 as) negates much of the sensitivity improvements that the Antarctic atmosphere offers, compared to mid-latitude sites such as Mauna Kea or Cerro Paranal. The seeing at mid-latitude sites, though smaller in amplitude, is dominated by turbulence at altitudes of $10-20 \mathrm{~km}$. Over the Antarctic Plateau, virtually no high altitude turbulence is present in the winter. The mean square error for an astrometric measurement with a dual-beam, differential astrometric interferometer in the very narrow angle regime is proportional to the integral of $h^{2} C_{N}^{2}(h)$. Therefore, sites at which the turbulence occurs only at low altitudes offer large gains in astrometric precision. We show that a modest interferometer at the South Pole can achieve $10 \mu$ as differential astrometry 300 times faster than a comparable interferometer at a good mid-latitude site, in median conditions. Science programs that would benefit from such an instrument include planet detection and orbit determination, and astrometric observation of stars microlensed by dark matter candidates.
\end{abstract}

Keywords: instrumentation: interferometers - techniques: interferometric - astrometry - extrasolar planets - Galaxy: halo, stellar content

\section{Introduction}

Properties of the atmosphere are the ultimate limit to astronomical observations from ground-based sites. Atmospheric transparency, background and seeing (indicated by the full width of the point spread function at half maximum, FWHM) are the parameters by which sites are typically compared. For observations in the optical and near infrared, high mountain sites that are above most of the Earth's boundary layer such as Mauna Kea and the Chilean Andes are superior because of favourable values of these parameters. Transparency and background are parameters that are relatively simple to quantify and interpret. However, there is much more to seeing than the FWHM of images taken through the turbulent atmosphere.

The optical significance of turbulence in the atmosphere is due to refractive index fluctuations, driven by temperature fluctuations. These temperature fluctuations are usually described in terms of a Kolmogorov model, with a temperature structure function as follows:

$$
D_{T}(r)=\left\langle(T(x)-T(x+r))^{2}\right\rangle=C_{T}^{2} r^{2 / 3} .
$$

Here, $T$ is the temperature, $x$ and $r$ are location variables, and $C_{T}^{2}$ characterises the amplitude of the turbulence. These temperature fluctuations cause refractive index $(N)$ fluctuations through the refractivity dependence of air on temperature, $C_{N}^{2}=(\delta N / \delta T)^{2} C_{T}^{2}$.

The optical effect is characterised by the threedimensional refractivity power spectrum, which for Kolmogorov turbulence is

$$
\Phi(\kappa, h)=0.033 C_{N}^{2}(h) \kappa^{-11 / 3} .
$$

Here, $\kappa$ is the spatial frequency of the turbulence, and $C_{N}^{2}(h)$ describes the vertical profile, where $h$ is the height above the observatory. $C_{N}^{2}(h)$ is typically complicated, with multiple layers resulting from wind shear in the atmosphere.

The Fried parameter, $r_{0}$, relates the seeing to the equations above, and is the scale over which the heightintegrated phase fluctuation at a given wavenumber, $k=2 \pi / \lambda$, equals one radian $\mathrm{rms}$.

Given a $C_{N}^{2}(h)$ profile, the Fried parameter is

$$
r_{0}=\left[0.423 k^{2} \sec (z) \int C_{N}^{2}(h) d h\right]^{-3 / 5},
$$

and the FWHM of an image is $0.98 \lambda / r_{0} . z$ is the zenith angle of observation. For the rest of this paper, we will refer all calculations to the zenith.

Thus, the integrated amplitude of $C_{N}^{2}$ determines $r_{0}$ and therefore the seeing. The integrated seeing to ice level has proven to be a major drawback for Antarctic telescopes, where the seeing is $1-3$ as at $0.5 \mu \mathrm{m}$, a value more typical of poor, low-altitude sites than superb sites such as Mauna Kea or Cerro Paranal, where the seeing is routinely below an arcsecond.

However, other parameters, such as scintillation, isoplanatic angle, and tilt anisoplanatism, which have paramount significance in certain kinds of astronomical observations, depend on higher order moments of the $C_{N}^{2}$ profile. Specifically, tilt anisoplanatism is the limiting error term for astrometric interferometry, and is a result of the second moment of the $C_{N}^{2}$ profile. The mean square error for an astrometric measurement with a dual-beam, differential astrometric interferometer in the very narrowangle regime $(\theta h \ll B)$ with long integrations, $t \gg B / V$, 
is (Shao \& Colavita 1992):

$$
\sigma_{\delta}^{2}=5.25 B^{-4 / 3} \theta^{2} \int h^{2} C_{N}^{2}(h)(V t)^{-1} d h .
$$

In this equation, $B$ is the baseline of the interferometer, $\theta$ is the angular separation of the celestial objects, $V$ is the wind speed as a function of height, and $t$ is total integration time of the observation. This calculation assumes a Kolmogorov power spectrum with no outer scale. Due to the $h^{2}$ factor, this integral is completely dominated by high altitude turbulence. Thus, a site where the $C_{N}^{2}$ profile is devoid of high altitude turbulence provides a substantial advantage.

The South Pole is such a site. The $C_{N}^{2}(h)$ profile of the atmosphere above the South Pole is fundamentally different from that of any other characterised site (Marks et al. 1999). The unusual polar atmosphere is entirely dominated by low altitude turbulence and is not affected by the turbulence generating jet streams or trade winds nor the high-altitude synoptic wind shear that creates turbulence at mid-latitudes.

First, we explore the benefits of a South Pole interferometer quantitatively, and then highlight two scientific projects for such an interferometer.

\section{Benefits of a South Pole Interferometer}

The atmospheric turbulence at a site like Mauna Kea is dominated by high altitude turbulence generated by tropopause instability, jet streams, and wind shear between synoptic weather systems. The lack of solar stratospheric heating at the South Pole during the polar winter results in a nearly adiabatic upper atmosphere, which means that the optical effects of turbulence (due to temperature fluctuations) are substantially reduced. The tropopause is at least a factor of two lower and very weak. Furthermore, there are no jet streams or trade winds at the South Pole, which are responsible for the large wind shears observed in atmospheric profiles at mid-latitude sites.

In order to compare the sites, we describe the $C_{N}^{2}(h)$ profile in terms of a Hufnagel-Valley (HV) model (Hardy 1998; Roggemann \& Welsh 1996). The HV model is an empirically fitted heuristic model, used extensively in the context of theoretical calculations of light propagation through the turbulent atmosphere. The $C_{N}^{2}$ profile is fitted by a series of terms: a planetary boundary layer term of the form $C_{N}^{2}(h)=A_{1} \exp \left(-h / H_{1}\right)$, a tropopause term of the form $C_{N}^{2}(h)=A_{2} h^{10} \exp \left(-h / H_{2}\right)$, and an individual layer term of the form $C_{N}^{2}(h)=A_{3} \exp \left(-\left(h-H_{3}\right)^{2} / 2 d^{2}\right)$. The amplitudes, $A_{n}$, heights, $H_{n}$, and the thickness, $d$, of the third component are all determined empirically. The values of these parameters used in our models of Mauna Kea and the South Pole are indicated in Tables 1 and 2.

Since the astrometric error is affected by the wind speed, we model the atmospheric wind profile following Greenwood (1977), but we neglect the zenith angle and azimuth terms. This model consists of aconstant ground

Table 1. Mauna Kea atmosphere model (Hardy 1998)

\begin{tabular}{lllll}
\hline Term & $\begin{array}{c}\text { Boundary layer } \\
A \exp (-h / H)\end{array}$ & \multicolumn{1}{c}{$\begin{array}{c}\text { Tropopause } \\
A h^{10} \exp (-h / H)\end{array}$} & $\begin{array}{c}\text { Strong layer } \\
A \exp \left(-(h-H)^{2} / 2 d^{2}\right)\end{array}$ & Total \\
\hline$A$ & $1 \times 10^{-17} \mathrm{~m}^{-2 / 3}$ & $1.6 \times 10^{-53} \mathrm{~m}^{-2 / 3} \mathrm{~m}^{-10}$ & $1 \times 10^{-16} \mathrm{~m}^{-2 / 3}$ & \\
$H(\mathrm{~m})$ & 3000 & 1000 & 6500 & 20 \\
$d(\mathrm{~m})$ & & & 1000 & $0.5^{\prime \prime}$ \\
$r_{0}(\mathrm{~cm})$ & 65 & 44 & 27 & 100 \\
FWHM & $0.15^{\prime \prime}$ & $0.23^{\prime \prime}$ & 69 & 69 \\
$\sigma_{\delta}(\mu \mathrm{as})$ & 23 & 69 & & \\
\hline
\end{tabular}

The contributions of individual terms are calculated, in addition to the total. The Fried parameter, $r_{0}$, is calculated at $0.5 \mu \mathrm{m}$. The total $r_{0}$ is calculated as $r_{0}=\left(\sum_{i} r_{0_{i}}^{-5 / 3}\right)^{-3 / 5}$. FWHM $=0.98 \lambda / r_{0}$. The calculated atmospheric astrometric error, $\sigma_{\delta}$, is the root mean square error for a $100 \mathrm{~m}$ baseline interferometer in $1 \mathrm{~h}$ on stars separated by 1 arcmin, neglecting all non-atmospheric noise sources. $\sigma_{\delta}$ adds in quadrature.

Table 2. South Pole atmosphere model in winter adapted from Marks et al. (1999)

\begin{tabular}{lllcc}
\hline Term & $\begin{array}{c}\text { Lower boundary layer } \\
A \exp (-h / H)\end{array}$ & $\begin{array}{c}\text { Upper boundary layer } \\
A \exp (-h / H)\end{array}$ & $\begin{array}{c}\text { Strong layer } \\
A \exp \left(-(h-H)^{2} / 2 d^{2}\right)\end{array}$ & Total \\
\hline$A$ & $2 \times 10^{-15} \mathrm{~m}^{-2 / 3}$ & $2 \times 10^{-15} \mathrm{~m}^{-2 / 3}$ & $2 \times 10^{-14} \mathrm{~m}^{-2 / 3}$ & \\
$H(\mathrm{~m})$ & 100 & 400 & 30 & 5.5 \\
$d(\mathrm{~m})$ & & & 40 & $1.8^{\prime \prime}$ \\
$r_{0}(\mathrm{~cm})$ & 21 & 36 & 6 & 6 \\
FWHM & $0.48^{\prime \prime}$ & $0.27^{\prime \prime}$ & 2 & $1.6^{\prime \prime}$ \\
$\sigma_{\delta}(\mu \mathrm{as})$ & 2 & 5 & 2 & \\
\hline
\end{tabular}

The terms and notation are the same as in Table 1. 
layer term and a Gaussian profile tropopause wind term:

$$
V(h)=V_{\text {ground }}+V_{T} \exp \left[-\left(\frac{h-H}{T}\right)^{2}\right]
$$

For the Mauna Kea model, we use $V_{\text {ground }}=5 \mathrm{~m} \mathrm{~s}^{-1}$, $V_{T}=25 \mathrm{~m} \mathrm{~s}^{-1}, H=12 \mathrm{~km}, T=5 \mathrm{~km}$. For the South Pole model, we use a higher ground wind speed, and a more moderate tropopause speed at a lower altitude: $V_{\text {ground }}=10 \mathrm{~m} \mathrm{~s}^{-1}, V_{T}=10 \mathrm{~m} \mathrm{~s}^{-1}, H=7 \mathrm{~km}, T=3 \mathrm{~km}$. The astrometric error is not strongly sensitive to the details of the wind speed.

Marks et al. $(1996,1999)$ have measured the $C_{N}^{2}$ profile at the South Pole with tower and balloon based microthermal sensors. This profile clearly shows that the vast majority of the turbulence is at altitudes below $1 \mathrm{~km}$. Our HV model is fitted to the median $C_{N}^{2}$ profile data of Marks et al. (1999). Integrating the $\mathrm{HV}$ model described in Table 2 and Figure 2 results in seeing of 1.8 as at $0.5 \mu \mathrm{m}$, typical for the site as measured by direct imaging and differential image motion monitors.

We now use the model to assess the astrometric errors. In order to compare sites and our calculations with others in the literature, we refer all calculations to an interferometer with a $100 \mathrm{~m}$ baseline, reference stars separated by 1 arcmin and $1 \mathrm{~h}$ integration, neglecting non-atmospheric noise sources.

For the South Pole model, the astrometric error is $\sigma_{\delta}=6 \mu$ as. We have also integrated a standard $0.5^{\prime \prime}$ seeing Mauna Kea model (Hardy 1998) in Table 1 and Figure 1. The astrometric error for the same interferometer parameters is $\sigma_{\delta}=100 \mu$ as. Another commonly used reference atmosphere for Mauna Kea is that of Roddier et al. (1990), which is the reference atmosphere used by Shao \& Colavita (1992). Integrating this $0.3^{\prime \prime}$ seeing profile we obtain an astrometric error of $\sigma_{\delta}=64 \mu \mathrm{as}$, comparable to the Shao \& Colavita (1992) value of $\sigma_{\delta}=60 \mu \mathrm{as}$, for a $100 \mathrm{~m}$ baseline and a star separation of $1 \mathrm{arcmin}$. This mode of interferometry has been demonstrated to achieve these levels of precision with the Mark III and Palomar Testbed Interferometer (Colavita 1994; Colavita et al. 1999).

A measure of performance is the integration time required to achieve a certain level of accuracy given the same interferometer configuration. Equation 1 indicates that the integration time is inversely proportional to $\sigma_{\delta}^{2}$. Therefore, these calculations indicate that an astrometric interferometer at the South Pole will achieve a given astrometric accuracy 300 times faster. A 300-fold increase in the efficiency of observation enables a number of science programs generally thought to be restricted to space-based interferometers.

This calculation is possibly optimistic. A few balloon measurements of $C_{N}^{2}$ (Marks et al. 1999) indicate that lowlevel high altitude turbulence appears at times during the Antarctic winter. This turbulence is enough to degrade the relative speed to a factor of between 4 and 10 compared to

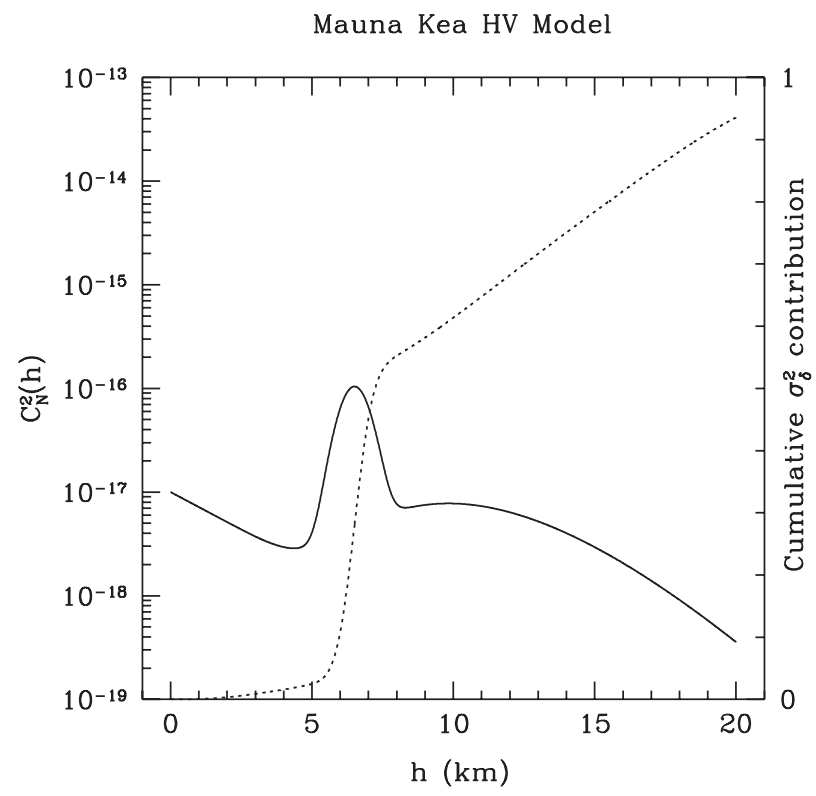

Figure 1 Mauna Kea atmosphere model (Hardy 1998). The solid line is the model $C_{N}^{2}(h)$ turbulent atmospheric profile. The dotted line shows the cumulative contribution to the mean square atmospheric astrometric error: $\sigma_{\delta}^{2}(h) / \sigma_{\delta}^{2}=\int_{0}^{h} h^{\prime 2} V\left(h^{\prime}\right)^{-1} C_{N}^{2}\left(h^{\prime}\right) d h^{\prime} /$ $\int_{0}^{\infty} h^{\prime 2} V\left(h^{\prime}\right)^{-1} C_{N}^{2}\left(h^{\prime}\right) d h^{\prime}$.

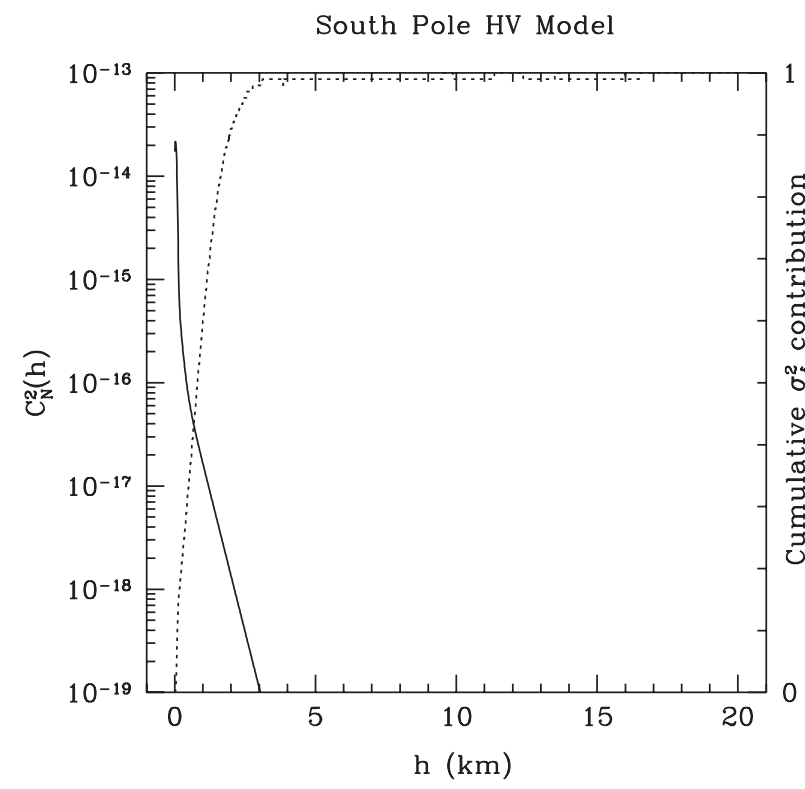

Figure 2 South Pole atmosphere model in winter adapted from Marks et al. (1999). The terms and notation are the same as in Figure 1.

Mauna Kea. However, this is a comparison of poor conditions at the South Pole to median or better at Mauna Kea. Furthermore, the high altitude turbulence measured by the balloon flights may be contaminated by wake turbulence from the balloon itself (Marks et al. 1999). Although this hardly affects the integrated seeing, the $h^{2}$ weighting for anisoplanatism strongly emphasises the high altitude turbulence. Further studies are warranted, particularly with SCIDAR measurements, which are most sensitive to high altitude turbulence and are immune to wake turbulence. 
There are two independent, though circumstantial, pieces of evidence that a large gain in astrometric error can be realised. First, Marks et al. (1999) calculates that the mean scintillation index, $\sigma_{I}^{2}$, at the South Pole is four tenths of the value at Cerro Paranal. Since the scintillation index depends on a $h^{5 / 6}$ moment of the $C_{N}^{2}$ profile, we can estimate a lower limit to the speed improvement compared to Paranal as $0.4^{-12 / 5}=9$.

Second, the tilt anisoplanatism term is similar to the Strehl anisoplanatism term that determines coherence over angular separations. Many people know the adage, 'Stars twinkle. Planets don't.' This is generally true because planets are larger than the isoplanatic angle at most locations on the Earth, so the scintillations of different parts of the planet disk are incoherent, and average out. If the isoplanatic angle is larger than the disk of a planet, then the planet will twinkle. During the South Pole winters of 1995 and 1996, one of us (JPL) saw Jupiter scintillate on occasion, indicating an isoplanatic angle larger than 30 as in the visible, even at an elevation of 22 degrees above the horizon. The mean conditions profile in Figure 2 gives an isoplanatic angle of 33 as at $0.5 \mu \mathrm{m}$ at the zenith. Since the isoplanatic angle is proportional to airmass to the $8 / 5$ power, this naked eye observation indicates that the zenith isoplanatic angle must be as large as 2 arcmin under some conditions. This is compelling evidence that the atmosphere above the Antarctic Plateau is uniquely lacking high altitude turbulence.

The mechanism that generates the existing turbulence at the South Pole is wind shear across the strongly inverted lower atmosphere. Such conditions are likely to exist anywhere in the Antarctic Plateau, but the strength and height of the inversion, and windspeed vary markedly. The height of the inversion layer at the South Pole strongly correlates with wind speed, since the settling of cold air into a strong, shallow inversion is disrupted by high wind. Wind over the Antarctic Plateau is dominated by katabatic flow from the high points of the plateau, downhill towards the coast. Therefore, higher sites such as Dome C, where the mean windspeed is only $2.6 \mathrm{~m} \mathrm{~s}^{-1}$, are likely to show strong, shallow thermal inversions, confining the turbulence generated to even lower altitudes, which will offer even larger gains to astrometric interferometry. Likely to be the ultimate site is the highest point on the plateau, Dome A. At these sites it is even possible that the turbulent inverted layer may be shallow enough that it is feasible to elevate the telescope above it, and realise the 'super seeing' suggested by Gillingham (1993). Site testing programs are underway to characterise these sites, including seeing monitoring programs. Measurements of the $C_{N}^{2}$ profiles will be crucial to determination of the scientific programs and instruments to be deployed at these sites.

\section{Science Applications}

The benefits shown in this calculation are limited to differential astrometric observations. Such observations have many applications. We highlight two of them here, which are particularly promising with regard to important astrophysical questions and well suited to be conducted in the southern hemisphere. We have also considered other configurations to take advantage of the unique properties of the $C_{N}^{2}(h)$ profile.

The factor of 300 in speed achievable with an interferometer at the South Pole result from the low altitude of the turbulence causing common aberrations that can be removed with a differential measurement. The same technique can be exploited in different ways with single telescopes and adaptive optics or interferometers. The large isoplanatic angle at the South Pole is attractive for adaptive optics applications. However, the small $r_{0}$ requires very bright guide stars and a high order AO system, even for a moderate sized telescope. Similarly, a conventional interferometer measuring fringe visibility suffers from the poor seeing. A dual star interferometer is required to gain an advantage from the low altitude of the turbulence with phase referencing, taking advantage of the coherence of the atmosphere across large angles. Such an interferometer can be used to measure fringe visibility on a faint source, while tracking on a bright source. A generally useful interferometer in this mode would require a large number of telescopes and long baselines to allow bootstrapping the phase referencing to long baselines. To measure relative fringe phase, however, only requires a single baseline, and it is disadvantageous to resolve the source, so moderate baselines are desirable. We therefore conclude that astrometric interferometry is uniquely suited to the site characteristics and logistical constraints in Antarctica.

\subsection{Planet Detection and Orbit Determination}

Astrometric observations with accuracies near a few microarcseconds permit the detection and determination of the orbits of extrasolar planets. For reference, if a star like the Sun, lying at a distance of $10 \mathrm{pc}$, has a Jupiter-like planet orbiting it, the star's position will be perturbed by a maximum of 1 mas over the orbital period $(\sim 12 \mathrm{yr})$. The signature of an Earth-like planet is approximately $1 \mu$ as. Because the South Pole interferometer could, in principle, conduct observations indefinitely, within $10 \mathrm{yr}$ one could not only survey and determine the three-dimensional orbital characteristics of the planets discovered through radial velocity studies, but also survey a substantial number of other stars within $30 \mathrm{pc}$ for signatures of extrasolar planets. By comparison, the Space Interferometry Mission (SIM), which has a limited lifetime of $5 \mathrm{yr}$, will be unable to observe stars over such long periods, limiting the types of planets it will find. It is necessary to fit the mass, semi-major axis, eccentricity, parallax and proper motion of the star in the astrometric solution. Accurate determination of all of these parameters with less than a complete orbit is not possible. It will therefore require significantly longer temporal baselines than SIM can provide to fully characterise the distribution of planets.

In comparison to the Keck Interferometer, the advantages of the South Pole interferometer are the factor of 300 
in speed of observation, meaning that many more stars could be surveyed in a given time. Alternatively, the substantially larger isoplanatic angle permits observation of more stars. For example, instead of speed, the benefits result in a 300-fold increase in the area of sky from which reference stars can be chosen.

\subsection{Astrometric Measurement of OGLE Microlensing Events}

There is a controversial body of evidence that suggests that a substantial fraction of the dark matter in the Milky Way is in the form of old white dwarfs. These may have been detected by microlensing experiments (MACHO; Alcock et al. (2000)) and direct surveys (Oppenheimer et al. 2001). However, the locations of the lensing masses remain unmeasurable and are a major source of contention among various researchers. It is possible that none of the microlensing events correspond to lenses in the Galaxy's halo. Astrometry of the source star during a microlensing event breaks the degeneracy in the solution to the microlensing equations. As a result, one can determine the mass and distance to the microlens itself. As shown by Boden, Shao, \& van Buren (1998), astrometric accuracy of better than $10 \mu$ as permits accurate determination of the lens parameters, although some degeneracy remains concerning the transverse velocity of the lens. Indeed, a lens placed at a distance of $8 \mathrm{kpc}$ with the source star in the Large Magellanic Cloud (LMC) produces an astrometric effect of about $100 \mu$ as, readily detectable with a modest baseline South Pole interferometer.

Although the MACHO project (Alcock et al. 2000) is no longer searching for microlenses, the OGLE-II project (Udalski, Kubiak, \& Szymanski 1997) is currently engaged in more sensitive observations of the LMC and their system for broadcasting early detection of new events is almost operational. The LMC is easily observed from the South Pole. With continuous observation possible during the polar night, an interferometer at the South Pole could resolve the remaining question concerning the nature of the MACHO objects.

\section{Conclusions}

We have demonstrated that the unique structure of the atmosphere above the high Antarctic Plateau provides significant advantages to interferometric astrometry. The calculations described above motivate improved measurements of the $C_{N}^{2}$ profile of the atmosphere with statistical coverage of varying conditions. If such measurements confirm our results, the construction of an interferometer at the South Pole should be considered for the near future. The observations by such an instrument will have a substantial impact on several important astrophysical problems.

\section{Acknowledgments}

JPL has been supported in part by the National Science Foundation Science and Technology Center for Adaptive Optics, managed by the University of California at Santa Cruz under cooperative agreement No. AST-9876783. We thank the University of California-Berkeley Center for Integrative Planetary Studies for a travel grant. BRO is supported by a Hubble Postdoctoral Research Fellowship (grant number HF-01122.01-99A). We thank Jean Vernin for providing electronic versions of and discussions of South Pole $C_{N}^{2}$ profiles and Geoff Marcy and Ben Lane for useful discussions. This work would not have been possible without the dedicated work of Rodney Marks, who died at the South Pole while wintering over.

\section{References}

Alcock, C., et al. 2000, ApJ, 542, 281

Boden, A. F., Shao, M., \& van Buren, D. 1998, ApJ, 502, 538

Colavita, M. M. 1994, A\&A, 283, 1027

Colavita, M. M., et al. 1999, ApJ, 510, 505

Gillingham, P. 1993, in Optics in Astronomy: 32nd Herstmonceux Conference, ed. J. V. Wall (Cambridge: Cambridge University Press), 244

Greenwood, D. P. 1977, J. Opt. Soc. Am., 67, 390

Hardy, J. W., ed. 1998, Adaptive Optics for Astronomical Telescopes (New York: Oxford University Press)

Marks, R. D., Vernin, J., Azouit, M., Briggs, J. W., Burton, M. G., Ashley, M. C. B., \& Manigault, J. F. 1996, A\&AS, 118, 385

Marks, R. D., Vernin, J., Azouit, M., Manigault, J. F., \& Clevelin, C. 1999, A\&AS, 134, 161

Oppenheimer, B. R., Hambly, N. C., Digby, A. P., Hodgkin, S. T., \& Saumon, D. 2001, Science, 292, 698

Roddier, F., Cowie, L., Graves, J. E., Songaila, A., \& McKenna, D. 1990, Proc. SPIE, 1236, 485

Roggeman, M. C., \& Welsh, B. 1996, Imaging Through Turbulence (CRC Press)

Shao, M., \& Colavita, M. M. 1992, A\&A, 262, 353

Udalski, A., Kubiak, M., \& Szymanski, M. 1997, Acta Astronom., 47, 319 